\title{
PERFORMANCE ANALYSIS OF DISTANCE TRANSFORM BASED INTER- SLICE SIMILARITY INFORMATION ON SEGMENTATION OF MEDICAL IMAGE SERIES
}

\author{
Eşref Selvi, Merve Özdemir and M. Alper Selver \\ Department of Electrical and Electronics Engineering, Dokuz Eylül University, 35160 \\ Kaynaklar Kampusu, Buca, Izmir, Turkey \\ alper.selver@deu.edu.tr
}

\begin{abstract}
Segmentation of organs from CT and MR image series is a challenging research area in all fields of medical imaging. Although, organs of interest are threedimensional in nature, slice-by-slice approaches are widely used in clinical applications because of their ease of integration with the current manual segmentation scheme (i.e. gold standard). Moreover, the high anisotropy of CT and MR data makes intra-slice information more reliable than inter-slice features. Nevertheless, slice-by-slice techniques should be supported with adjacent slice information since it is shown that features using the similarity of adjacent image slices outperform measures based on single-slice features in all cases. One of this similarity features is the distance transform which is shown to be effective on providing inter-slice similarity of abdominal organs. A parameter that control the vicinity of search area using the distance transform is $\alpha$, which determines the order of the power of distance transforms applied to the image. Since there is no study discussing the effect of $\alpha$ on segmentation performance, the aim of this study is to analyze how changes on $\alpha$ affects performance in terms of accuracy, computation and time requirements. The simulations performed on several medical image series and for four different abdominal organs show the importance of parameter analysis for distance transformation.
\end{abstract}

Key Words- Segmentation, Medical Image, Distance Transform, Classification

\section{INTRODUCTION}

State of art modalities in medical imaging (i.e. Computed Tomography (CT), Magnetic Resonance Imaging (MRI) etc.) can provide very large number of image slices with very small slice thickness (i.e. inter-slice distance) to effectively represent acquired part of the body [1]. Segmentation of organs from medical image series is a very important step of vital analysis prior to several clinical procedures. Extracting a body part of interest (i.e. organ, tissue, tumor etc.) via segmentation from these slices of images is an emerging field due to the trade-off between obtaining the efficiency in terms of the time requirements and the overall segmentation performance [2].

Current gold standard in clinic is slice-by-slice manual delineation of image series by using advanced software tools. However, this is a time consuming and tedious task due to the excessive amount of image slices. Therefore developing precise segmentation algorithms is an ongoing field of research, in which several semi-automatic and automatic techniques are introduced frequently. 
There are mainly two approaches for segmenting a series of medical images:

1) Three dimensional (3-D) approaches which use 3-D information provided by volumetric data constructed by integration of image slices; [2]

2) Slice-by-slice (or iterative 2-D) approaches which perform segmentation on a single image but use adjacent slice information in some manner $[3,4,6]$.

Although, organs of interest are three-dimensional in nature, the use of first approach (i.e. 3-D models and/or features) require a large number of training shapes/data not desirable for objects that can vary largely (i.e. most of the organs). Moreover, the high anisotropy of CT and MRI data makes intra-slice information more reliable than interslice features, which must be extracted after an interpolation process. Moreover, the results of 3-D algorithms are also in 3-D, preventing easy transition to 2-D images using which experts can make final modifications on segmentation result.

The latter approach (i.e. slice-by-slice analysis) has the advantage of complete integration with the current manual segmentation scheme. These methods should combine 2-D segmentation with appropriate slice similarity features. If an effective similarity measure is used, such methods would be computationally feasible for large number of slices and the drawbacks of a complete 3-D approach can be avoided [2,6].

Recently, the distance transform is introduced as an effective, yet computationally simple feature to represent slice similarity in abdominal datasets [6]. The distance transform provides a metric that measures the separation of the pixels in an image. The metric is calculated to measure a pre-defined (i.e. Euclidean etc.) distance along the horizontal, vertical, and diagonal directions. Thus, it provides information about an organ location at the adjacent (preceding/succeeding) slice by assuming the organ size and location does not change dramatically between adjacent slices. This is a reasonable assumption as emerging technology of medical modalities (i.e. CT, MRI) enables very small slice thicknesses. Distance transform may also be seen as a way of mimicking the decision process of an expert who shall follow tissue properties at preceding/succeeding the slices by revisiting them in case of unclear organ shape in an image slice.

In other words, the distance transform allows the usage of adjacent slice information as it limits the search area to a region that is slightly bigger than the previously segmented region. Although, it is used successfully in segmentation processes [6], the parameters of the transform and their effects on performance have not been discussed yet.

Thus, this study aims to measure the performance of a medical image segmentation algorithm for abdominal organs under varying distance transform parameters. Abdominal image series are very appropriate for the adjacent slice similarity measures due to the appearance of organs (i.e. liver, kidneys, spleen etc.) through the image series [3]. These organs have small size in the initial slices where they begin to appear, slightly expand in the successive ones and finally disappear with a gradual decrease in size o appearance [4]. Each organ of interest in these dataset (i.e. liver, kidneys, spleen etc.) can dominate a number of slices but eventually disappears. These changes in shape and size of the organs make abdominal studies a suitable data for adjacent slice similarity analysis.

The rest of the paper is organized as follows: Next section introduces the data used in this study. The third section describes methods used, while the fourth section covers the results obtained by application of these methods. Finally, the fifth section represents conclusions and discussions. 


\section{DATASETS and ORGANS OF INTEREST}

Our CT data sets (i.e. Fig. 1) were acquired after contrast agent injection at portal phase using a Philips Secura CT with two detectors and a Philips Mx8000 CTA with 4 detectors, both equipped with the spiral CT-Angiography option and located in Dokuz Eylül University Radiology Department. Our MRI data sets (i.e. Fig. 2) were acquired using a 1,5 Tesla MRI system located at the same department. Both CT and MRI images are 12 bit DICOM images. CT images have a resolution of $512 \times 512$ while the resolution of MRI data is $256 \times 256$. The data sets were chosen randomly from the Picture Archiving and Communication System (PACS). Adjacent CT images used in this study have $3.2 \mathrm{~mm}$ slice thickness while this value is $2 \mathrm{~mm}$ for MRI.

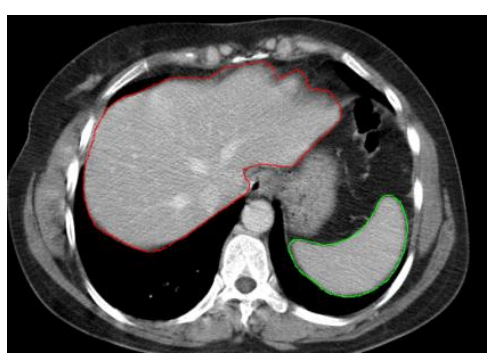

(a)

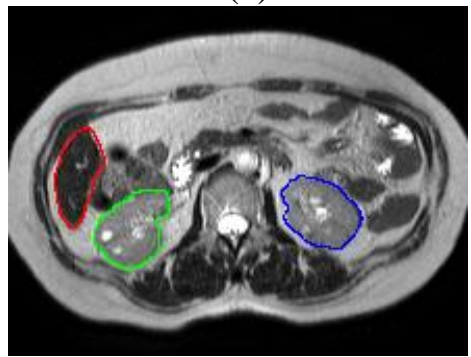

(d)

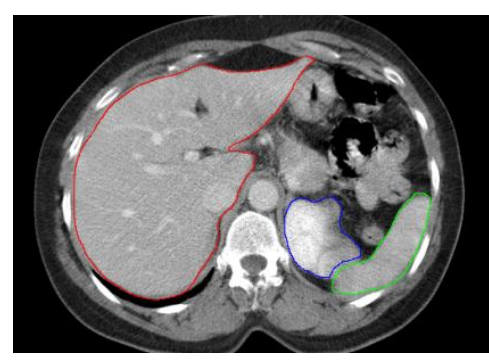

(b)

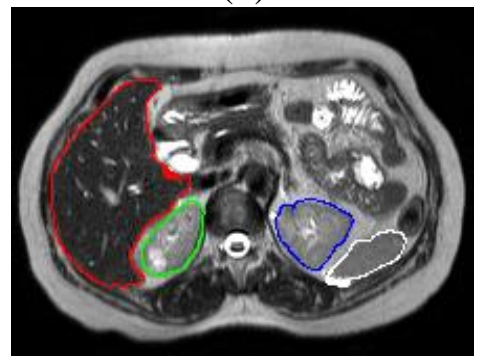

(e)

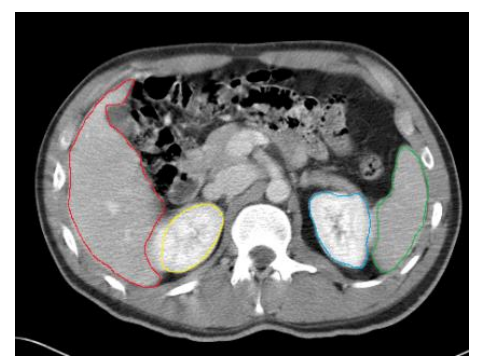

(c)

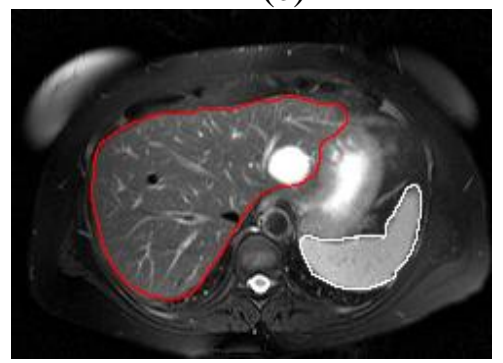

(f)

Figure 1. (a-c) CT image slices from different parts of the body, (d-f) MR image slices from different parts of the body

The abdominal organs of interest (i.e. liver, right/left kidneys, spleen), their basic properties in a bottom-to-top acquired CTA series and their inter-relations with each other can be summarized as follows: Taking the spine, which is located at the bottom middle, the liver is located at the right side of the body (left side of the images due to the direction of acquisition), at upper right of the right kidney, and it is adjacent to the right kidney and spleen. The size of liver is large and its size and shape differ significantly through image slices. Additionally, its parenchyma texture (excluding inner vasculature) and HU range is almost the same as spleen (Fig 1.a) for CT but such a correlation does not exist for MRI (Fig. 1.f).

The two kidneys are located at the both sides of the spine (Fig. 1.c and Fig. 1.d). Their shape is usually circular but their location may differ from patient to patient. They are very small at their first time of appearance in the images and therefore conflict with the boundary of other organs, especially the liver. The spleen is located at the left side of the image. It is adjacent to the left kidney and the liver if the liver has a typical shape. 
One can comment on the overall sizes of these organs (i.e. liver is the biggest, spleen is bigger than the kidneys etc.) but at each slice their appearance changes relative to each other (see Fig. 1). For instance when the liver is reduced to a small object, the spleen and the kidneys might appear bigger than the liver (Fig 1.d).

The appearances and existence of these organs in an image slice have countless variations and this requires the use of very adaptive and robust features to obtain adjacent slice similarity information.

\section{METHODOLOGY}

\subsection{The Distance Transform}

The distance transform provides a metric or measure of the separation of points in the image. This transform gives the distance transform of image, in which the value of each pixel is replaced by its distance to the nearest background pixel. Distance transform gives an image object in which the value of each pixel is the absolute Euclidean distance in pixels to the nearest pixel of background.

In 2-D, the Euclidean distance between $\left(x_{1}, y_{1}\right)$ and $\left(x_{2}, y_{2}\right)$ are coordinates of the image.

$$
d=\sqrt{\left(x_{1}-x_{2}\right)^{2}+\left(y_{1}-y_{2}\right)^{2}}
$$

To illustrate the effect of distance transform in an image, a matrix is constructed whose elements are ones and zeros as seen in Fig. 2.a. The value of one represents an object; the value of zero represents background. This matrix can be considered as a binary image. Euclidean distance transform of the binary image is computed as seen in Fig. 2.b. For each pixel in the binary image, the distance transform assigns a number that is equal to the distance between that pixel and the nearest non-zero pixel of binary image. To increase the effect of the distance transform, different orders of power of distance transformation are computed in this study. In Fig. 2.c and Fig. 2.d, second order and third order powers of the distance transformation are applied to the binary image, respectively. It can be easily observed that after the distance transform, nearest pixels have small values thanks to Euclidean distance transform formula. Pixels, which are far from object pixels, have larger values and in the light of this knowledge, when the order of the power of the distance transformation is increased, the pixels that are distant from the object have much higher values than the pixels that are nearer (i.e. See Fig. 2.c and Fig. 2.d.

In our algorithm, distance transform is applied to MR and CT images with respect to different organs. In Figure 3.a-3.d, the distance transform is applied to MR images, which are obtained in same dataset, and the order of power of the distance transform is fixed to a constant value. 


\begin{tabular}{|l|l|l|l|l|l|l|l|l|l|}
\hline 0 & 0 & 0 & 0 & 0 & 0 & 0 & 0 & 0 & 0 \\
\hline 0 & 1 & 0 & 0 & 0 & 0 & 0 & 0 & 0 & 0 \\
\hline 0 & 1 & 1 & 0 & 0 & 0 & 0 & 0 & 0 & 0 \\
\hline 0 & 1 & 1 & 1 & 0 & 0 & 0 & 0 & 0 & 0 \\
\hline 0 & 1 & 1 & 1 & 1 & 0 & 0 & 0 & 0 & 0 \\
\hline 0 & 1 & 1 & 1 & 0 & 0 & 0 & 0 & 0 & 0 \\
\hline 0 & 1 & 1 & 0 & 0 & 0 & 0 & 0 & 0 & 0 \\
\hline 0 & 1 & 0 & 0 & 0 & 0 & 0 & 0 & 0 & 0 \\
\hline 0 & 0 & 0 & 0 & 0 & 0 & 0 & 0 & 0 & 0 \\
\hline 0 & 0 & 0 & 0 & 0 & 0 & 0 & 0 & 0 & 0 \\
\hline
\end{tabular}

(a)

\begin{tabular}{|c|c|c|c|c|c|c|c|c|c|}
\hline 2 & 1 & 2 & 5 & 8 & 13 & 18 & 25 & 32 & 41 \\
\hline 1 & 0 & 1 & 2 & 5 & 8 & 13 & 18 & 25 & 34 \\
\hline 1 & 0 & 0 & 1 & 2 & 5 & 8 & 13 & 20 & 29 \\
\hline 1 & 0 & 0 & 0 & 1 & 2 & 5 & 10 & 17 & 26 \\
\hline 1 & 0 & 0 & 0 & 0 & 1 & 4 & 9 & 16 & 25 \\
\hline 1 & 0 & 0 & 0 & 1 & 2 & 5 & 10 & 17 & 26 \\
\hline 1 & 0 & 0 & 1 & 2 & 5 & 8 & 13 & 20 & 29 \\
\hline 1 & 0 & 1 & 2 & 5 & 8 & 13 & 18 & 25 & 34 \\
\hline 2 & 1 & 2 & 5 & 8 & 13 & 18 & 25 & 32 & 41 \\
\hline 5 & 4 & 5 & 8 & 13 & 18 & 25 & 32 & 41 & 50 \\
\hline
\end{tabular}

(c)

\begin{tabular}{|l|l|l|l|l|l|l|l|l|l|}
\hline 1,41 & 1,00 & 1,41 & 2,24 & 2,83 & 3,61 & 4,24 & 5,00 & 5,66 & 6,40 \\
\hline 1,00 & 0,00 & 1,00 & 1,41 & 2,24 & 2,83 & 3,61 & 4,24 & 5,00 & 5,83 \\
\hline 1,00 & 0,00 & 0,00 & 1,00 & 1,41 & 2,24 & 2,83 & 3,61 & 4,47 & 5,39 \\
\hline 1,00 & 0,00 & 0,00 & 0,00 & 1,00 & 1,41 & 2,24 & 3,16 & 4,12 & 5,10 \\
\hline 1,00 & 0,00 & 0,00 & 0,00 & 0,00 & 1,00 & 2,00 & 3,00 & 4,00 & 5,00 \\
\hline 1,00 & 0,00 & 0,00 & 0,00 & 1,00 & 1,41 & 2,24 & 3,16 & 4,12 & 5,10 \\
\hline 1,00 & 0,00 & 0,00 & 1,00 & 1,41 & 2,24 & 2,83 & 3,61 & 4,47 & 5,39 \\
\hline 1,00 & 0,00 & 1,00 & 1,41 & 2,24 & 2,83 & 3,61 & 4,24 & 5,00 & 5,83 \\
\hline 1,41 & 1,00 & 1,41 & 2,24 & 2,83 & 3,61 & 4,24 & 5,00 & 5,66 & 6,40 \\
\hline 2,24 & 2,00 & 2,24 & 2,83 & 3,61 & 4,24 & 5,00 & 5,66 & 6,40 & 7,07 \\
\hline
\end{tabular}

(b)

\begin{tabular}{|c|c|c|c|c|c|c|c|c|c|}
\hline 2,83 & 1,00 & 2,83 & 11,18 & 22,63 & 46,87 & 76,37 & 125,00 & 181,02 & 262,53 \\
\hline 1,00 & 0,00 & 1,00 & 2,83 & 11,18 & 22,63 & 46,87 & 76,37 & 125,00 & 198,25 \\
\hline 1,00 & 0,00 & 0,00 & 1,00 & 2,83 & 11,18 & 22,63 & 46,87 & 89,44 & 156,17 \\
\hline 1,00 & 0,00 & 0,00 & 0,00 & 1,00 & 2,83 & 11,18 & 31,62 & 70,09 & 132,57 \\
\hline 1,00 & 0,00 & 0,00 & 0,00 & 0,00 & 1,00 & 8,00 & 27,00 & 64,00 & 125,00 \\
\hline 1,00 & 0,00 & 0,00 & 0,00 & 1,00 & 2,83 & 11,18 & 31,62 & 70,09 & 132,57 \\
\hline 1,00 & 0,00 & 0,00 & 1,00 & 2,83 & 11,18 & 22,63 & 46,87 & 89,44 & 156,17 \\
\hline 1,00 & 0,00 & 1,00 & 2,83 & 11,18 & 22,63 & 46,87 & 76,37 & 125,00 & 198,25 \\
\hline 2,83 & 1,00 & 2,83 & 11,18 & 22,63 & 46,87 & 76,37 & 125,00 & 181,02 & 262,53 \\
\hline 11,18 & 8,00 & 11,18 & 22,63 & 46,87 & 76,37 & 125,00 & 181,02 & 262,53 & 353,55 \\
\hline
\end{tabular}

(d)

Figure 2. (a) A binary image (b) distance transform of the binary image (c) second order power of the distance transform of the binary image (d) third order power of the distance transform of the binary image

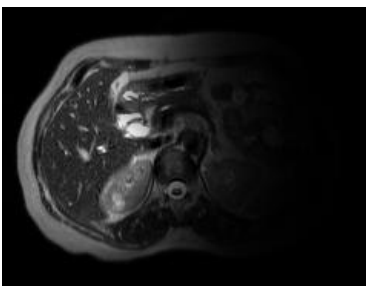

(a)

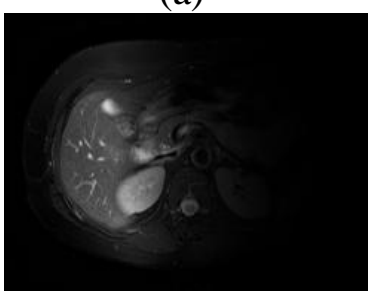

(e)

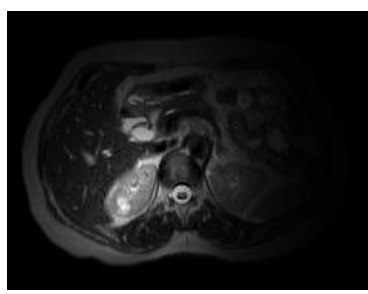

(b)

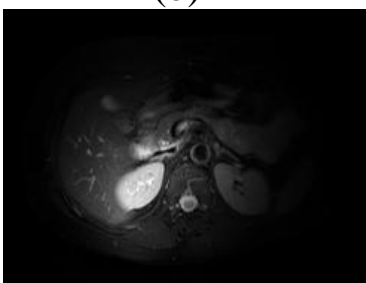

(f)

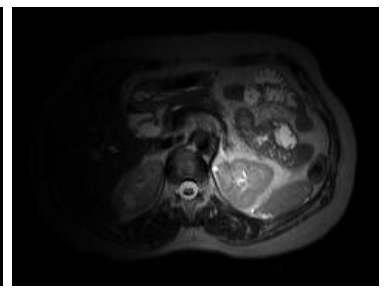

(c)

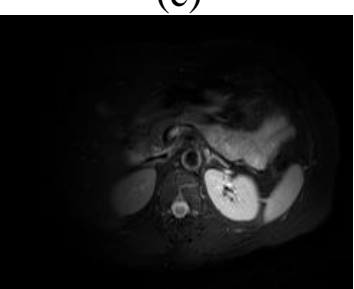

(g)

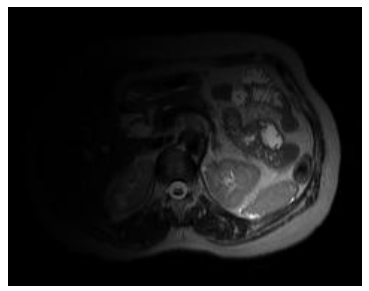

(d)

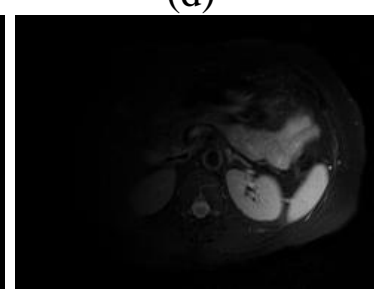

(h)

Figure 3. Examples of abdominal MR images with same order of distance with respect to: (a)liver; (b) right kidney; (c) left kidney; and (d) spleen.

For different organs, the response of distance transform is observed according to organs and the same procedure is repeated for different MR dataset and CT datasets (Fig. 3 (e- 
h) and Fig. 4 (a-h)). In case of the absence of an organ in a slice, (i.e. Fig. 4.b), the response of the distance transform of that slice becomes an empty matrix.

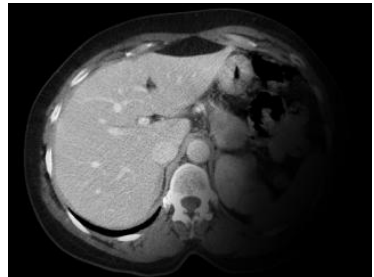

(a)

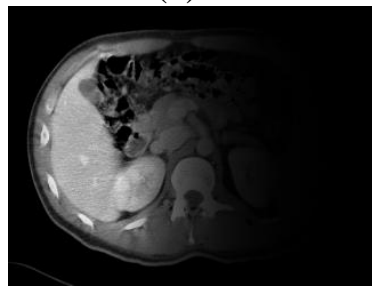

(e)

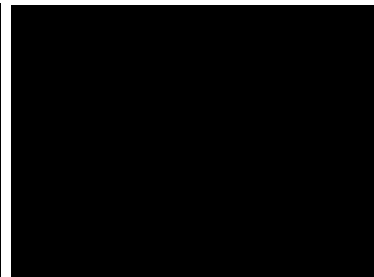

(b)

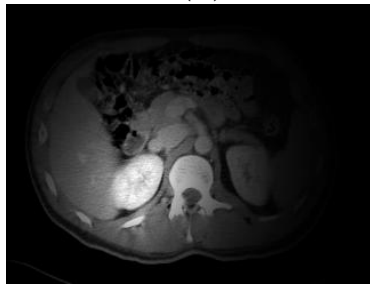

(f)

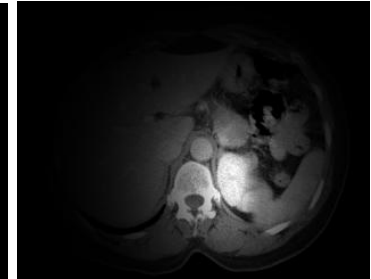

(c)

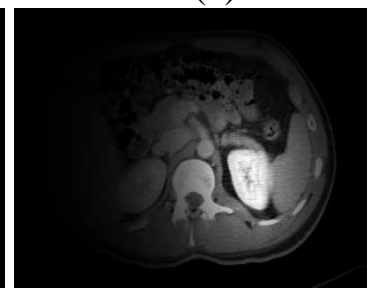

(g)

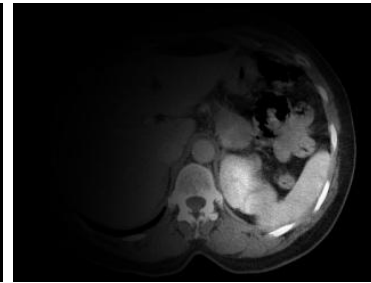

(d)

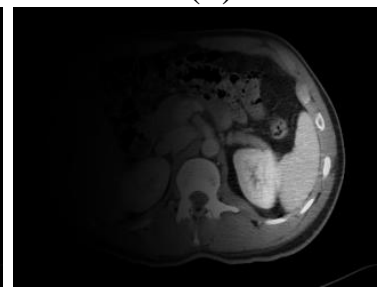

(h)

Figure 4. Examples of abdominal CT images with same order of distance with respect to: dataset 1: (a) liver; (b) right kidney; (c) left kidney; and (f) spleen; dataset 1: (e) liver; (g) right kidney; (h) left kidney; and (i) spleen.

In this paper, our main goal is to indicate the effects for different orders of distance transform in MR and CT images. For instance, in a CT slice, the order effects are observed in the right kidney and shown in Figure 5.a-5.c. By increasing the order of the distance, right kidney is obtained more evident.

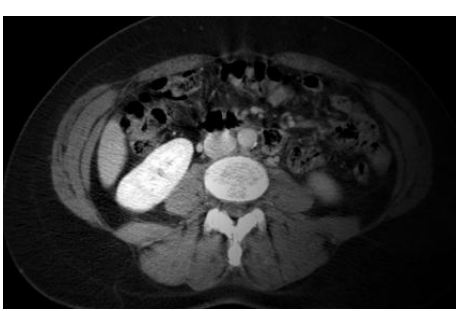

(a)

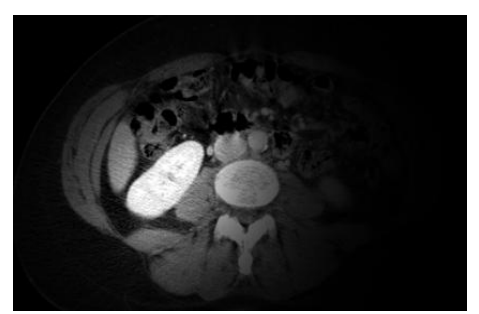

(b)

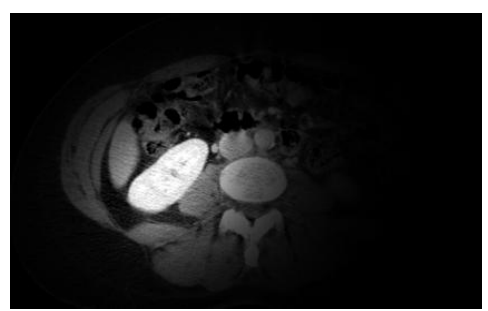

(c)

Figure 5. Examples of abdominal CT images with different order of distance in the right kidney (a) order $=2$; (b) order $=5$; (c) order $=7$.

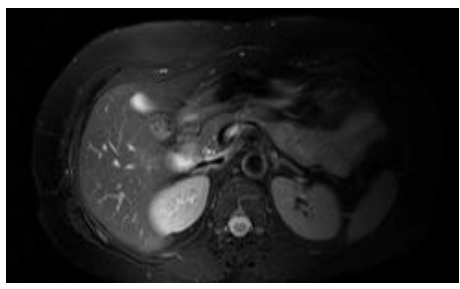

(a)

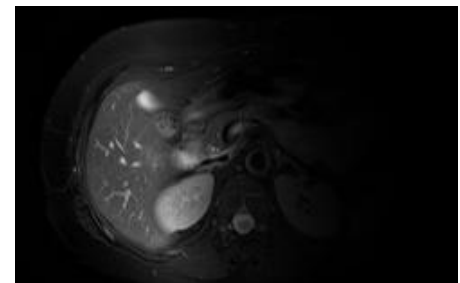

(b)

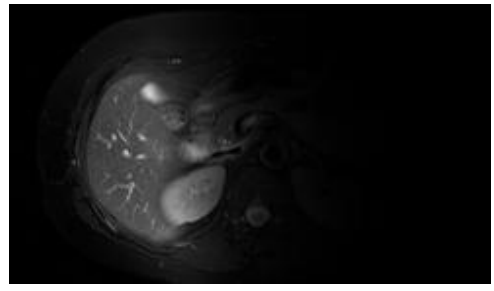

(c)

Figure 6. Examples of abdominal MR images with different order of distance in the liver (a) order $=2$; (b) order $=5$; (c) order $=7$.

In Figure 6, Figure 7 and Figure 8, different order responses of distance transform are obtained according to four organs in different MR and CT datasets. 


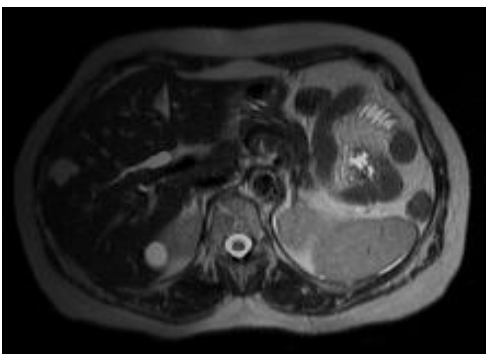

(a)

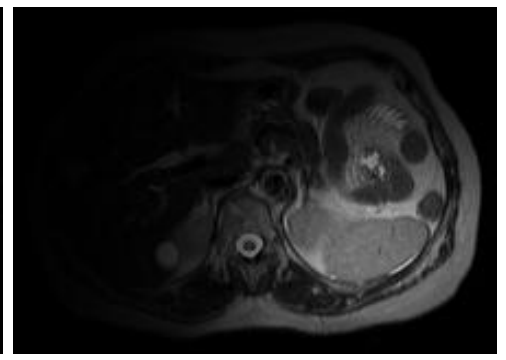

(b)

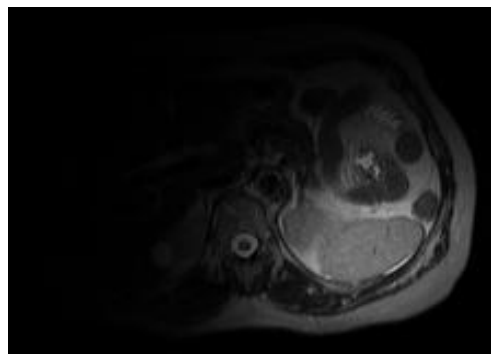

(c)

Figure 7. Examples of abdominal MR images with different order of distance in the spleen (a) order $=2$; (b) order $=5$; (c) order $=7$.

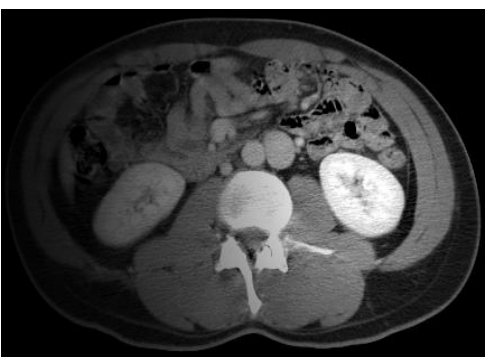

(a)

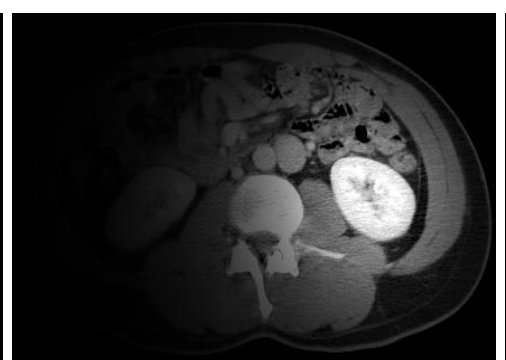

(b)

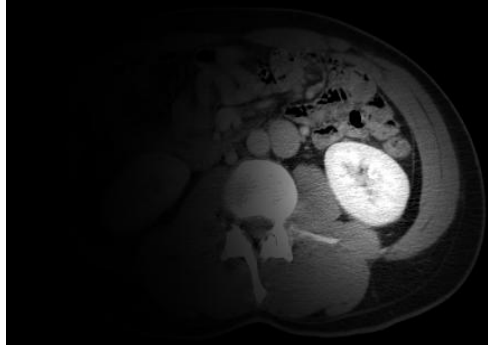

(c)

Figure 8. Examples of abdominal CT images with different order of distance in the left kidney (a) order $=2$; (b) order $=5$; (c) order $=7$.

\subsection{Segmentation Algorithm}

In our algorithm, a starting slice, which is segmented manually (i.e. Labeled starting slice), is obtained. Distance transform of the labeled starting slice is multiplied by next slice to obtain down segmentation in the image dataset. In a similar manner, distance transform of starting slice is multiplied by previous slice to obtain up segmentation in the image dataset. The block diagram of the procedure is shown in Figure 9. Initializing with the segmented image of the starting slice and by using distance transform in down and up segmentations, transformed images, which will be used as the input of feature extraction and then segmentation, are obtained (Fig. 5-7). This procedure is applied continuously until all images are processed for down and up segmentation.

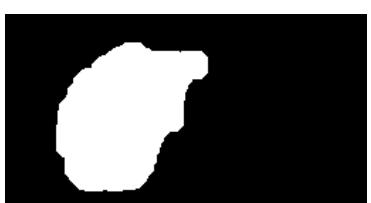

a)

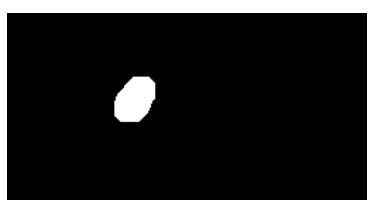

(b)

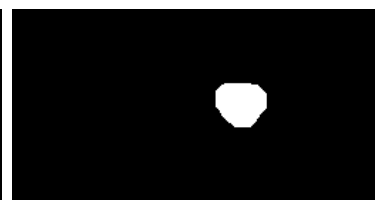

(c)

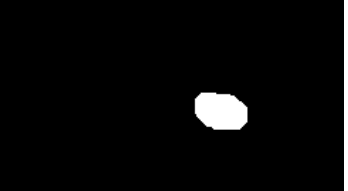

(d)

Figure 10. Classification algorithm results for different organs (a) Liver; (b) Left kidney; (c) Right kidney; (d) Spleen 


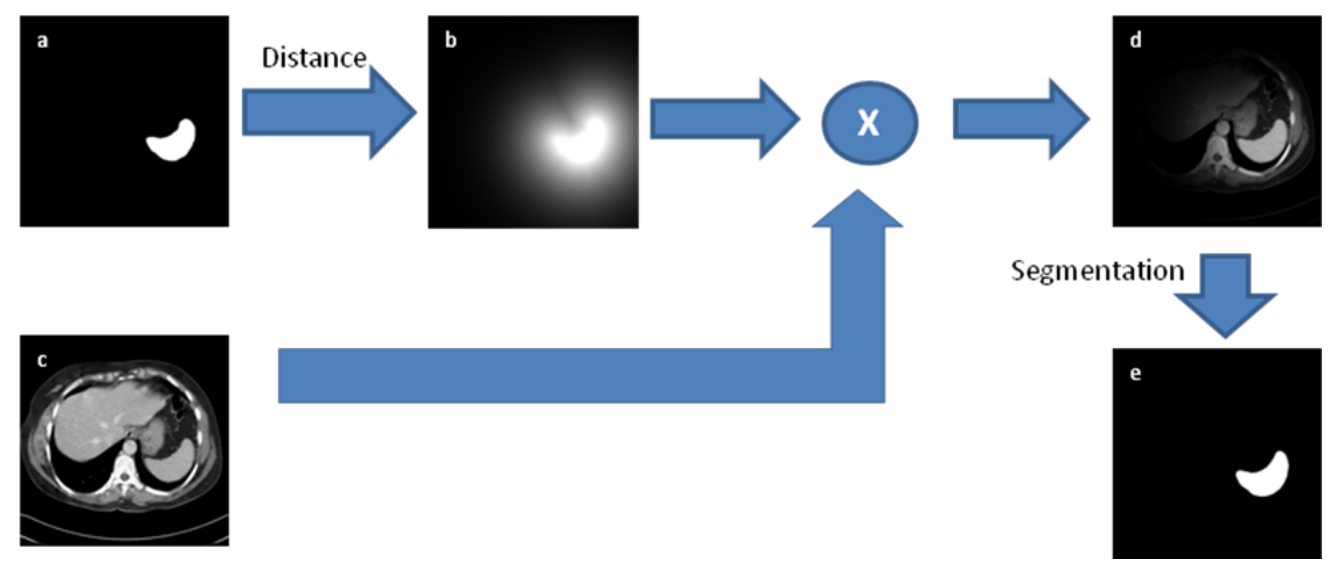

Figure 9. (a) Previously segmented organ (from previous or next slice) (b) distance transform of slice in a (c) current slice to segment the organ (d) dot product of the slice in $\mathrm{b}$ and $\mathrm{c}$ (e) segmented organ with post-processing

From the transformed images, classification based segmentation is performed by extracting features followed by classifying them (Fig. 10.). Finally, after application of the distance transform and segmentation, to obtain a better segmented image, morphological operators are used (Fig. 11.) [5].
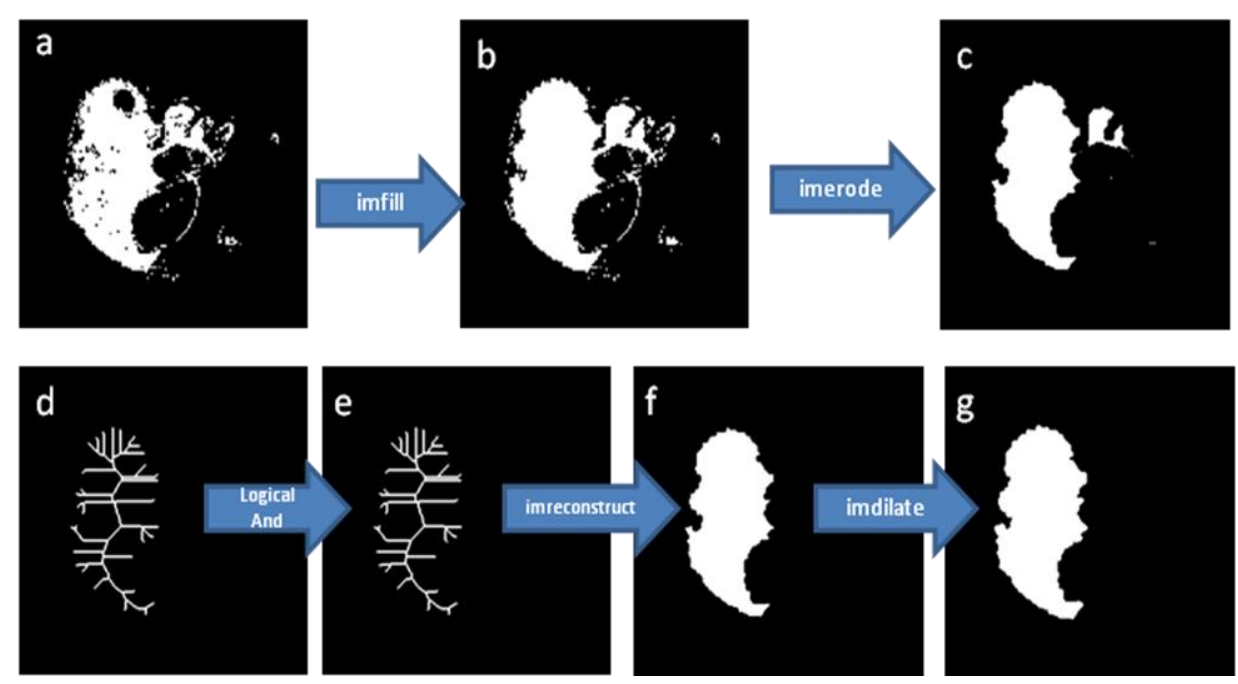

Figure 11: a) Post-processing of a slice; results of: (a) segmentation algorithm result (b) filling the holes c)erosion d) skeletonization segment liver of the previous slice e) logical "AND" to image in c and d (f) constructing image using image in e as mask and image in $\mathrm{c}$ as the marker $\mathrm{g}$ ) dilation to restore contours ( $\mathrm{g}$ ) dilating the image in $\mathrm{f}$

\section{SIMULATIONS RESULTS}

Several simulations are performed to determine how the order of distance transformation affects the segmentation performance. Some examples of these results are presented in Tables 1 and 2. The metrics chosen for evaluating performance during 
these simulations are CC: Correct classification, FP: False Positives, FN: False negatives, SE: Sensitivity, SP: Specificity, PPV: Positive predictive value and NPV: Negative predictive value. If $\mathrm{N}=\mathrm{TP}+\mathrm{TN}+\mathrm{FP}+\mathrm{FN}$; where $\mathrm{TP}$ : true positives and $\mathrm{TN}$ : True negatives, the percentage formulas of these metrics can be given as per_CC $=100 *(\mathrm{TP}+\mathrm{TN}) . / \mathrm{N} ; \quad$ per_FP $=100 * \mathrm{FP} . /(\mathrm{TN}+\mathrm{TP}) ; \quad$ per_FN $=100 * \mathrm{FN} . /(\mathrm{TP}+\mathrm{FN})$; per_SE $=100 * \mathrm{TP} . /(\mathrm{TP}+\mathrm{FN})$; per_SP $=100 * \mathrm{TN} . /(\mathrm{TN}+\mathrm{FP})$; per_PPV=100*TP./(TP+FP); per_NPV=100*TN./(TN+FN).

Table 1. Metrics and run time of different slices in two CT datasets

\begin{tabular}{|c|c|c|c|c|c|c|c|c|}
\hline$\alpha$ & \multicolumn{7}{|c|}{ CT2 } & 14 th slice spleen metrics \\
\cline { 2 - 9 } & CC & FP & FN & SE & SP & PPV & NPV & run time (s) \\
\hline 2,00 & 99,85 & 0,02 & 15,29 & 84,71 & 99,98 & 97,03 & 99,87 & 1499,69 \\
\hline 5,00 & 99,81 & 0,04 & 18,51 & 81,49 & 99,96 & 95,00 & 99,84 & 1487,79 \\
\hline 7,00 & 99,73 & 0,04 & 27,67 & 72,33 & 99,96 & 94,51 & 99,76 & 1501,82 \\
\hline
\end{tabular}

(a)

\begin{tabular}{|c|c|c|c|c|c|c|c|c|}
\hline$\alpha$ & \multicolumn{7}{|c|}{ CT1 21th slice liver metrics } \\
\cline { 2 - 9 } & CC & FP & FN & SE & SP & PPV & NPV & run time (s) \\
\hline 2,00 & 99,07 & 0,04 & 5,69 & 94,31 & 99,95 & 99,71 & 98,96 & 1575,05 \\
\hline 5,00 & 99,24 & 0,09 & 4,25 & 95,75 & 99,89 & 99,38 & 99,22 & 1515,09 \\
\hline 7,00 & 99,34 & 0,10 & 3,63 & 96,37 & 99,89 & 99,37 & 99,33 & 1522,03 \\
\hline
\end{tabular}

(b)

Table 2. Metrics and run time of different slices in two MRI datasets

\begin{tabular}{|c|c|c|c|c|c|c|c|c|}
\hline$\alpha$ & \multicolumn{6}{|c|}{ MR2 9th slice Right Kidney metrics } \\
\cline { 2 - 9 } & CC & FP & FN & SE & SP & PPV & NPV & run time (s) \\
\hline 2,00 & 99,91 & 0,05 & 5,94 & 94,06 & 99,95 & 93,29 & 99,96 & 1263,33 \\
\hline 5,00 & 99,87 & 0,06 & 9,63 & 90,37 & 99,94 & 91,49 & 99,93 & 1256,97 \\
\hline 7,00 & 99,92 & 0,07 & 1,43 & 98,57 & 99,93 & 91,10 & 99,99 & 1273,62 \\
\hline
\end{tabular}

(a)

\begin{tabular}{|c|c|c|c|c|c|c|c|c|}
\hline \multirow{2}{*}{$\alpha$} & \multicolumn{6}{|c|}{ MR1 17th slice liver metrics (without post-processing) } \\
\cline { 2 - 9 } & CC & FP & FN & SE & SP & PPV & NPV & run time (s) \\
\hline 2,00 & 94,47 & 2,01 & 48,03 & 51,97 & 97,94 & 67,42 & 96,14 & 1284,284 \\
\hline 5,00 & 95,06 & 2,02 & 39,90 & 60,10 & 97,92 & 70,29 & 96,77 & 1274,186 \\
\hline 7,00 & 95,05 & 2,06 & 39,50 & 60,50 & 97,88 & 69,99 & 96,80 & 1263,97 \\
\hline
\end{tabular}

(b) 
The results show that the order of power of distance transform has a varying effect on the performance. For instance, FN ratio increases as $\alpha$ increase in Table 1.a for spleen while FN ratio decreases as $\alpha$ decrease in Table 1.b for liver in another dataset.

\section{CONCLUDING REMARKS}

The distance transform is an effective feature for representing adjacent slice similarity in segmentation applications. The order of its power, $\alpha$, determines the search area for an organ of interest; however its effects on the performance have not been analyzed so far. In this study, it is shown that varying values of $\alpha$ have drastical effects on system performance as shown in exemplary applications presented in Table 1, Table 2 and Figure 12 (see spleen line). However, more simulations and further studies are required to determine the causes behind this effect and how to choose, $\alpha$, to obtain optimal performance.

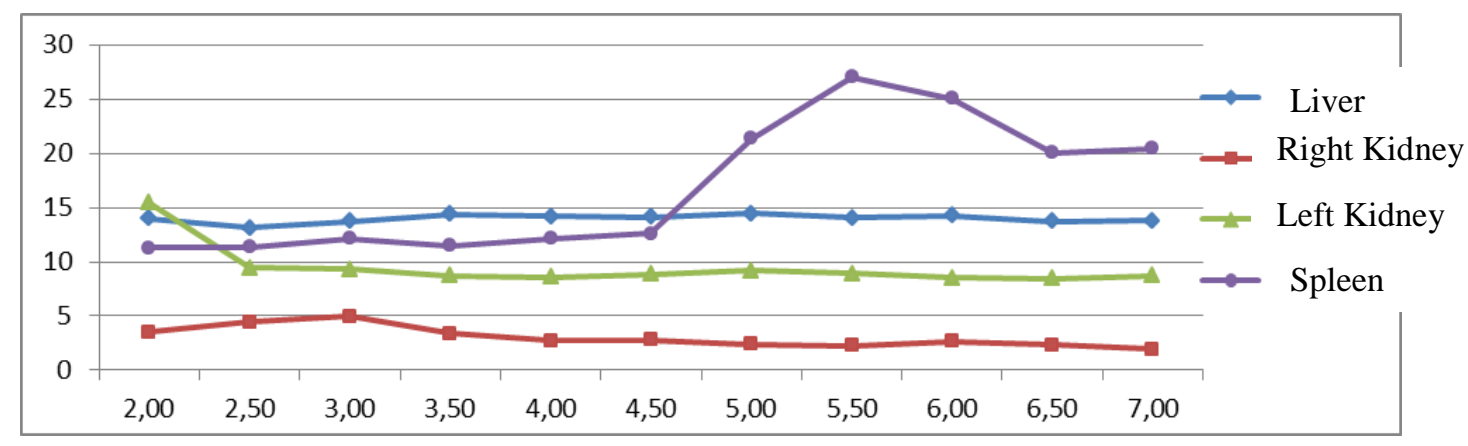

Figure 12. The order of the power of distance transformation vs. FN percentage graph. Spleen FN rate changes significantly for some values of $\alpha$.

\section{REFERENCES}

1. G. Flohr, S. Schaller, K. Stierstorfer, H. Bruder, B. M. Ohnesorge, Multi detector row CT systems and image reconstruction techniques, Radiology 235, 756-773, 2005.

2. L. Bidaut, Data and Image Processing for abdominal imaging, Abdominal Imaging, vol. 25, Springer Verlag, Berlin, 341-360, 2000.

3. J. E. Koss, F. D. Newman, T. K. Johnson, D. L. Kirch, Abdominal organ segmentation using texture transforms and a hopfield neural network, IEEE Transactions on Medical Imaging 18 (7) 640-648, 1999.

4. C. C. Lee, P. C. Chung, H. M. Tsai, Identifying multiple abdominal organs from CT image series using a multimodule contextual neural network and spatial fuzzy rules, IEEE Transactions on Information Technology in Biomedicine 7 (3), 208-217, 2003.

5. L. Vincent, Morphological grayscale reconstruction in image analysis: applications and efficient algorithms, IEEE Transactions on Image Processing 2(2) 176-201, 1993.

6. M. Alper Selver, A. Kocaoğlu, G . Demir , H. Doğan, O. Dicle, C. Güzeliş, Patient oriented and robust automatic liver segmentation for pre-evaluation of liver transplantation, Computers in Biology and Medicine 38(7), 765-784, 2008. 